1 Universidade Federal Fluminense (UFF), Instituto de Saúde Coletiva (ISC) Niterói (RJ), Brasil. pattyfidelis@id.uff.br

2 Universidade do Estado do Rio de Janeiro (Uerj), Instituto de Medicina Social (IMS) - Rio de Janeiro (RJ), Brasil.

${ }^{3}$ Fundação Oswaldo Cruz (Fiocruz), Escola Nacional de Saúde Pública Sergio Arouca (Ensp) - Rio de Janeiro (RJ), Brasil.

\section{Organização da Atenção Primária à Saúde em um município rural remoto do norte do Brasil}

\author{
Organization of Primary Health Care in a remote rural municipality \\ in northern Brazil
}

Karina Vasconcelos Rodrigues', Patty Fidelis de Almeida1, Lucas Manoel da Silva Cabral2, Márcia

Cristina Rodrigues Fausto ${ }^{3}$

DOI: $10.1590 / 0103-1104202113105$

\begin{abstract}
RESUMO O objetivo do artigo foi caracterizar a organização da Atenção Primária à Saúde (APS) e suas interfaces com os demais serviços da rede assistencial em um Município Rural Remoto (MRR). Foi realizado estudo de caso único em Assis Brasil (AC), por meio de entrevistas com usuários, gestores e profissionais de saúde. Os resultados indicaram distribuição desigual de estabelecimentos de saúde com áreas descobertas; dificuldades de acesso por condições climáticas; barreiras econômicas para custeio de transporte; promoção de ações itinerantes na zona rural; descontinuidade e insuficiência de medicamentos; dificuldades para a fixação de profissionais; escassez de recursos tecnológicos; falta de acesso à internet; necessidade de adaptação cultural; concentração de serviços especializados do SUS na capital. Foram identificados esforços da gestão local para manutenção da Estratégia Saúde da Família (ESF) e adequação dos processos de trabalho para atendimento ao grande fluxo de demanda espontânea, estrangeiros e população indígena. Argumenta-se que o MRR e suas populações somam vulnerabilidades econômicas, sociais e de acesso aos serviços de saúde, parcialmente atendidas pelas políticas nacionais, e que o ente municipal, sem o suficiente apoio e aporte de recursos estadual e federal, mantém arranjos possíveis para a provisão de APS, nem sempre afeitos aos princípios abrangentes da ESF.
\end{abstract}

PALAVRAS-CHAVE Atenção Primária à Saúde. Acesso aos serviços de saúde. Saúde da população rural. Ecossistema amazônico. Brasil.

ABSTRACT The aim of the article was to characterize the organization of Primary Health Care (APS) and its interfaces with other services in the healthcare network in a Remote Rural Municipality (MRR). A single case study was carried out in Assis Brasil ( $A C)$, through interviews with users, managers and health professionals. The results indicated an unequal distribution of health facilities with uncovered areas; access difficulties due to weather conditions; economic barriers to costing transport; promotion of itinerant actions in rural areas; discontinuity and insufficiency of medications; difficulties in retaining professionals; scarcity of technological resources; lack of internet access; need for cultural adaptation; concentration of specialized services of the Unified Health System (SUS) in the capital. Local management efforts were identified to maintain the Family Health Strategy (ESF) and the adequacy of work processes to meet the large flow of spontaneous demand, foreigners and the indigenous population. It is argued that the MRR and its populations add economic, social and access to health services vulnerabilities, partially covered by national policies, and that the municipal entity, without sufficient support and allocation of state and federal resources, maintains possible arrangements for the provision of APS, not always bound by the comprehensive principles of the ESF. 


\section{Introdução}

O acesso aos serviços de Atenção Primária à Saúde (APS) de alta qualidade representa a forma mais eficiente e equitativa de alcançar os melhores resultados em saúde'. No entanto, residentes em comunidades remotas ao redor do mundo têm acesso relativamente mais restrito aos cuidados primários ${ }^{2}$. Segundo a Organização Mundial da Saúde, cerca de 46\% da população mundial reside em áreas rurais remotas ${ }^{3}$, enquanto a maioria dos trabalhadores de saúde vive e trabalha nas médias e grandes cidades ${ }^{4}$.

Características dos territórios rurais remotos influenciam a estrutura, a função dos serviços de saúde, como os profissionais trabalham e a natureza das práticas ${ }^{5}$. Desigualdades socioeconômicas, pobreza e níveis mais baixos de emprego, juntamente com desequilíbrios relacionados com a disponibilidade de serviços e profissionais de saúde, infraestrutura das unidades de APS, transporte e comunicação entre as áreas rurais e centros urbanos e maior concentração dos recursos nas cidades, resultam em maior exclusão social dos moradores de áreas rurais ${ }^{6-8}$. Ao conjunto de vulnerabilidades socioeconômicas e ambientais mencionadas, observam-se também transformações expressivas nos modos de vida da população rural em função da dinâmica do agronegócio em algumas regiões ${ }^{8}$. Indicadores epidemiológicos expressam as desvantagens significativas para os Municípios Rurais Remotos (MRR) ${ }^{9}$ quando comparados à média para o Brasil, entre os quais: coeficiente de mortalidade infantil (MRR-17,56; Brasil-12,39); taxa de internações de alta complexidade (MRR-0,13; Brasil-0,44); percentual de nascidos vivos com pré-natal adequado (MRR-52,81; Brasil-70,76); e percentual de casos com início tardio do tratamento de câncer (MRR-21,7; Brasil-21,7) ${ }^{10}$.

Localidades com características rurais requerem serviços de saúde que atendam às necessidades de seus moradores ${ }^{11}$. Ao desenvolver uma política de saúde rural, os países enfrentam grandes desafios relativos a recursos humanos, governança, transporte, financiamento e comunicação ${ }^{12}$. Nesse sentido, estratégias e políticas diferenciadas para prestação de serviços de saúde em territórios rurais remotos vêm sendo implementadas por diversos países, como Austrália, Canadá, Nova Zelândia, Estados Unidos da América, países do Sudeste Asiático e do bloco Europeu12-14. No Brasil, destaca-se o esforço no campo da política de saúde de atender reivindicações dos movimentos sociais para a construção de uma política específica para os povos que representam a ruralidade brasileira - a Política Nacional de Saúde Integral das Populações do Campo, da Floresta e das Águas (PNSIPCFA), que orienta as bases para a organização dos serviços de saúde para estas populações.

No Brasil, a expansão da APS por meio das equipes da Estratégia Saúde da Família (ESF) colaborou para assegurar acesso mais resolutivo e humanizado à maioria da população ${ }^{15}$. No entanto, condições sociais e econômicas desfavoráveis das famílias e territórios, expressas na ausência de transporte público, escassez de serviços de saúde, falta de infraestrutura, como energia elétrica, saneamento básico e acesso a recursos tecnológicos e meios de comunicação ${ }^{16}$, imprimem maiores obstáculos e desafios à atuação das Equipes de Saúde da Família (EqSF). Tais condições, frequentemente encontradas em MRR brasileiros ${ }^{17}$, nem sempre são atendidas ou atenuadas por meio das diretrizes e padrões das políticas nacionais. As definições de espaço rural e urbano no Brasil foram redefinidas no ano de 201717, alinhadas a metodologias internacionais da Organização para a Cooperação e Desenvolvimento Econômico (OCDE) e da União Europeia. Localização em relação aos principais centros urbanos, densidade demográfica e tamanho da população estão entre os critérios para classificação dos municípios em cinco tipos: urbano; intermediário adjacente; intermediário remoto; rural adjacente; e rural remoto. De acordo com a nova classificação, $76 \%$ da população brasileira vive em áreas predominantemente urbanas $(26 \%$ 
dos municípios). Dos 5.570 municípios, 60,4\% foram classificados como predominantemente rurais, sendo $54,6 \%$ rurais adjacentes e $5,8 \%$ rurais remotos ${ }^{17}$.

O presente artigo, a partir da experiência e da percepção de gestores, profissionais e usuários, tem como objetivo caracterizar a organização da APS e suas interfaces com os demais serviços da rede assistencial em um MRR da região amazônica, buscando identificar especificidades e estratégias empreendidas que possam sinalizar caminhos para o aperfeiçoamento das práticas e políticas de saúde em contexto rural.

\section{Metodologia}

A metodologia adotada foi a do estudo de caso do tipo exploratório e instrumental ${ }^{18}$, com abordagem qualitativa, com vistas a produzir conhecimento sobre a organização da APS em um contexto ainda pouco explorado - MRR da região amazônica. Embora se trate de estudo de caso único - o município de Assis Brasil, pertencente ao estado do Acre, Brasil -, foi selecionado por ser 'típico'. Nesse sentido, acredita-se que represente, além da realidade amazônica, o contexto de municípios rurais transfronteiriços.

\section{Cenário do estudo}

O cenário de estudo foi constituído pelo MRR de Assis Brasil, localizado nos limites do território brasileiro, fazendo divisa com outros dois países, Peru e Bolívia, o que condicionava um padrão de grande circulação de pessoas. Embora mais da metade da população estivesse concentrada na sede municipal, era expressivo o quantitativo de residentes em áreas da zona rural, em geral, pequenos povoados dispersos e distantes da sede ${ }^{19}$. Parte da população era constituída por populações ribeirinha e por grande concentração de indígenas das etnias Jaminawá e Manchineri que vivem na Terra Indígena Mamoadate20. $\mathrm{O}$ município agregava áreas de proteção ambiental em seu território, como a Reserva Extrativista Chico Mendes, território marcado por extenso conflito de terras e desmatamento. Assis Brasil tem como característica peculiar a multiculturalidade, especialmente pela presença de povos tradicionais e pela condição de tripla fronteira internacional. A tabela 1 apresenta características do território e da população. Os dados mostram, na comparação com parâmetros estadual e nacional, que o município apresenta maiores vulnerabilidades relacionadas com renda, disponibilidade de bens públicos, pobreza, entre outras.

Tabela 1. Caracterização socioeconômicas da população, Município Rural Remoto, região Norte do Brasil, Assis Brasil, AC, 2019

\begin{tabular}{|c|c|c|c|}
\hline Municípios & & & \\
\hline Caracterização do Território & Assis Brasil & Acre $(A C)$ & Brasil \\
\hline População (hab.) ${ }^{1}$ & 6.072 & 733.559 & 190.732 .694 \\
\hline Expectativa populacional 2019 (hab.) $)^{2}$ & 7.534 & 894.470 & 210.147 .125 \\
\hline \% população rural ${ }^{3}$ & 38 & 33 & 16 \\
\hline \% da população beneficiária do PBF4 & 84 & 41 & 21 \\
\hline Área $\left(\mathrm{km}^{2}\right)^{1}$ & $4.974,174$ & $164.123,964$ & $8.510 .295,914$ \\
\hline Densidade demográfica (hab./ km²)1 & 1,22 & 4,47 & 22,43 \\
\hline Índice de Gini & 0,61 & 0,63 & 0,60 \\
\hline $\mathrm{IDHM}^{1}$ & 0,588 & 0,663 & 0,727 \\
\hline
\end{tabular}


Tabela 1. (cont.)

\begin{tabular}{|c|c|c|c|}
\hline Municípios & & & \\
\hline Caracterização do Território & Assis Brasil & Acre (AC) & Brasil \\
\hline Renda per capita $(R \$)^{1}$ & 291,33 & 522,15 & 793,87 \\
\hline Renda domiciliar menor que $1 / 2$ salário mínimo $(R \$)^{1}$ & 66,28 & 50,97 & 34,67 \\
\hline $\begin{array}{l}\text { \% da população em domicílios com banheiro e água } \\
\text { encanada? }\end{array}$ & 41 & 86 & 87 \\
\hline \% da população em domicílios com energia elétrica & 84 & 91 & 98 \\
\hline \% da população em domicílios com coleta de lixo & 59 & 34 & 97 \\
\hline$\%$ de extremamente pobres ${ }^{1}$ & 31 & 16 & 7 \\
\hline $\begin{array}{l}\text { Taxa de Analfabetismo - \% da população com } 15 \text { anos } \\
\text { ou mais }{ }^{1}\end{array}$ & 21 & 16 & 10 \\
\hline Área de Preservação Ambiental ${ }^{3}$ & $\begin{array}{r}1 \text { UCs [estação ecológica } \\
\text { rio acre]; } 2 \text { TIR [mamoada- } \\
\text { te e rio acre] } \\
\text { Reserva Extrativista Chico } \\
\text { Mendes }\end{array}$ & $\begin{array}{r}2 \text { Unidades de } \\
\text { Conservação } \\
\text { (UCs) e } 36 \text { TIR - } \\
47,9 \% \text { do terri- } \\
\text { tório protegido }\end{array}$ & $\begin{array}{r}2.229 \text { áreas } \\
\text { protegidas } \\
(3.884 .125 \\
\left.\mathrm{km}^{2}\right)\end{array}$ \\
\hline
\end{tabular}

Fonte: Elaboração própria.

${ }^{1}$ PNUD: Atlas do Desenvolvimento Humano no Brasil, Censo Demográfico - Competência, 2010.

2IBGE: Instituto Brasileiro de Geografia e Estatística, Expectativa da população - Competência, 2019.

3Mops: Mapas Estratégicos para Políticas de Cidadania - Competência, 2019.

${ }^{4}$ MDS: Ministério do Desenvolvimento Social, painel do Bolsa Família e Cadastro Único no seu município - Competência, 2019.

Programa Bolsa Família - PBF; Unidades de Conservação - UCs; Terras Indígenas Reconhecidas - TIR.

\section{População de estudo, instrumentos e coleta de dados}

Foram realizadas 16 entrevistas semiestruturadas em visita in loco ao município de Assis Brasil em maio de 2019. Os roteiros elaborados para cada tipo de informante-chave permitiram mapear a organização, a oferta e a disponibilidade de serviços de saúde no município, como também captar as nuances do trabalho e assistência em saúde no contexto rural remoto. Três grupos de informantes participaram do estudo: i) gestores (municipais, regionais e estaduais); ii) profissionais (Agentes Comunitários de
Saúde - ACS; enfermeiros e médico das ESF); e iii) usuários de três eventos traçadores ${ }^{21}$ definidos no estudo 22 para construção das trajetórias de cuidado: Câncer de Colo do Útero (CCU), Pré-natal, Parto e Puerpério (PPP) e Hipertensão Arterial Sistêmica (HAS). As entrevistas com profissionais e usuários foram realizadas em duas Unidades Básicas de Saúde (UBS), ambas localizadas na sede do município - uma responsável pela oferta de atendimento de população residente na sede do município, e outra, para a população residente em áreas afastadas da sede municipal (interior/zona rural). O perfil dos entrevistados é apresentado no quadro 1 . 
Quadro 1. Perfil dos entrevistados, Município Rural Remoto, região Norte, Assis Brasil, AC, 2019

Características

Assis Brasil (AC)

Total de entrevistados: 16

Gestores e Profissionais de Saúde [n=10]

Sexo'

Feminino

Masculino

20-29

$30-39$

40-49

50-59

Escolaridade / Formação'

Ensino médio

Enfermagem

Técnico Enfermagem

Medicina

Administração

Psicologia

Função

Gerente da divisão de APS (Gestor Estadual)

Gestor Regional

Secretário Municipal de Saúde

Coordenador do polo de Saúde Indígena

Enfermeiro EqSF

Médico EqSF

Agente Comunitário de Saúde

Tempo na função

Menos que 1 ano

4

Até 3 anos

Vínculo Trabalhista1

Estatutário

Celetista

Cargo Comissionado

Contrato temporário

Bolsista (PMM)

ios $[n=6]$

$\frac{\text { Usuá }}{\text { Sexo }^{1}}$

Feminino

4

Masculino

Idade $^{1}$

20-29

40-49

até 60

Fonte: Elaboração própria

'Banco de dados da pesquisa Atenção Primária à Saúde em Municípios Rurais e Remotos no Brasil.

\section{Análise dos dados}

A análise temática ${ }^{23}$ foi guiada por categorias inspiradas em estudos nacionais e internacionais sobre organização da APS ${ }^{24-26}$ e emergentes no campo: disponibilidade de serviços; território; ações desenvolvidas pelas equipes e no território; disponibilidade de medicamentos e realização de exames básicos; colaboração interprofissional; intersetorialidade; estratégias para garantia da força de trabalho em saúde; acessibilidade 
aos serviços de APS; serviços de saúde de referência para a APS.

A pesquisa 'Atenção Primária à Saúde em territórios rurais e remotos no Brasil' foi aprovada pelo Comitê de Ética em Pesquisa em Seres Humanos da Escola Nacional de Saúde Pública Sergio Arouca da Fundação Oswaldo Cruz, identificada pelo CAAE 92280918.3.0000.5240 e pelo parecer de aprovação $\mathrm{n}^{\circ} 2.832 .559$.

\section{Resultados}

\section{Organização da APS}

\section{DISPONIBILIDADE DE SERVIC̣OS E TERRITÓRIO}

Em Assis Brasil, a APS está organizada com base nas diretrizes nacionais da ESF. Eram 3 UBS, todas localizadas na sede municipal, nas quais estavam distribuídas 3 EqSF com 18 ACS, além de uma unidade definida como ponto de apoio no interior. Além das UBS em funcionamento, duas aguardavam inauguração (uma UBS na sede e um ponto de apoio próximo à área indígena). Todas as EqSF atuavam em UBS localizadas na sede municipal e estavam organizadas para atender usuários residente nos interiores já que não havia UBS nessas localidades. Na figura 1, é possível visualizar a concentração de UBS na sede municipal, inclusive o Polo de Saúde Indígena, e indisponibilidade de serviços de saúde no interior do município.

Embora se considerasse haver 100\% de cobertura potencial, havia áreas descobertas pela ESF, famílias não vinculadas à EqSF, principalmente em áreas isoladas e de difícil acesso. Médicos reforçavam que a vinculação à EqSF não era critério para definição do local de atendimento, sendo frequente o atendimento de usuários em UBS nas quais não estavam cadastrados, sem a garantia da continuidade do cuidado.

Figura 1. Distribuição das UBS e do Polo de Saúde Indígena, Município Rural Remoto, região Norte, Assis Brasil, AC, 2019

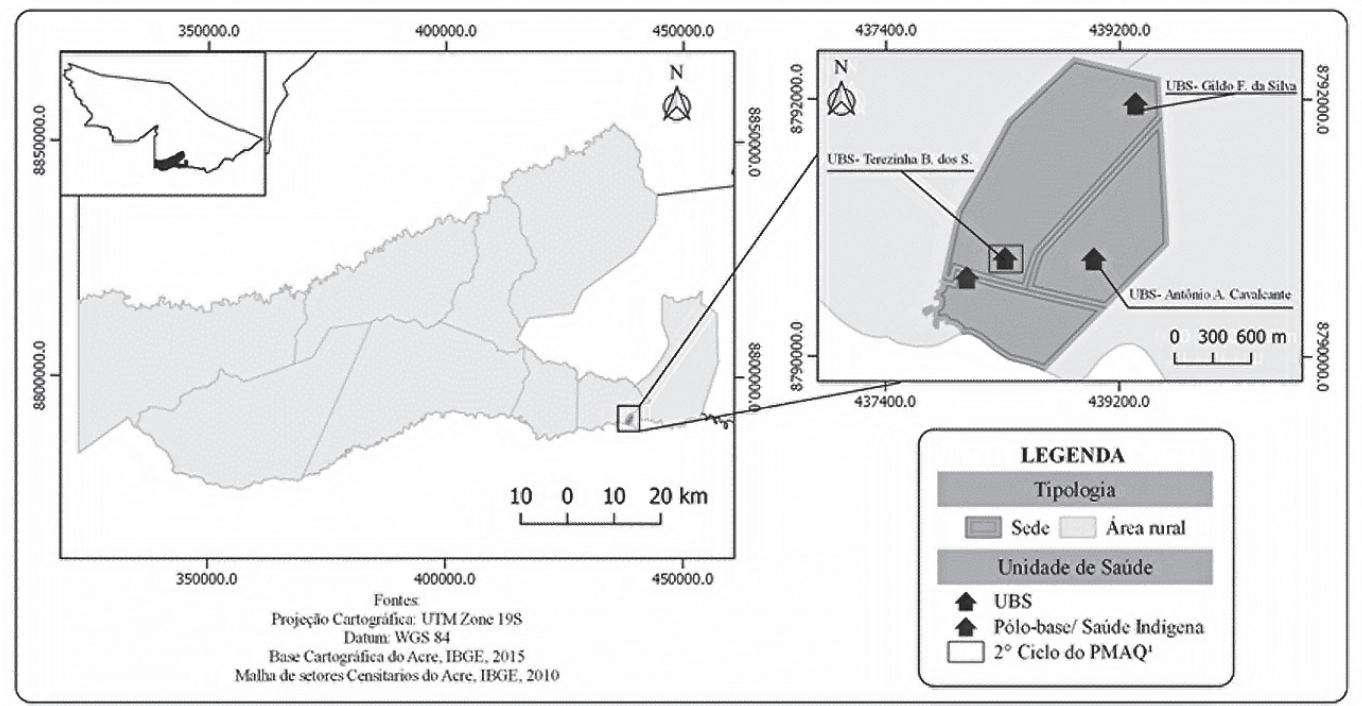

Fonte: Elaboração própria.

Nota: Unidades Básicas de Saúde (UBS) que participaram do segundo ciclo do Programa Nacional de Melhoria e da Qualidade da Atenção Básica (PMAQ-AB). 
O município dispunha de Academia da Saúde e Núcleo de Apoio à Saúde da Família (Nasf) ou, conforme termo atual, Núcleo Ampliado de Saúde da Família, com nutricionista, assistente social, psicólogos, farmacêutico, fisioterapeutas e educador físico. Havia também uma Unidade Mista Estadual com funcionamento 24 horas na qual eram realizados exames laboratoriais básicos, partos de risco habitual e exame radiológico. Médicos da APS faziam plantão na unidade mista com contratação temporária via Secretaria Municipal de Saúde (SMS). A tabela 2 , apresenta os recursos e equipamentos em saúde disponível no município.

A população indígena do município era expressiva e buscava atendimento tanto no subsistema de saúde indígena quanto no sistema de saúde municipal, ainda que não vinculada à ESF. O Distrito Sanitário Especial Indígena (DSEI) mantinha trabalho colaborativo com a SMS, no entanto, as ações voltadas para essa população eram desarticuladas e, por vezes, sobrepunham-se porque o acesso dessa população ao serviço de APS municipal era informal e não havia interação entre as equipes de APS dos dois sistemas de saúde. Os profissionais da ESF referiram dificuldades no atendimento aos indígenas, principalmente pela barreira cultural e de comunicação, sendo comum a presença de intérpretes do DSEI para viabilizar $\mathrm{o}$ atendimento.

A condição de município de tripla fronteira trazia enorme desafio para a gestão, organização e provisão da APS, flagrante nos relatos dos gestores e profissionais. A livre circulação entre os países propiciava, também, a circulação de agravos típicos da região. As atividades laborais exercidas por brasileiros do outro lado da fronteira, como prostituição e derrubada de árvores nativas da floresta amazônica, fomentavam a transmissão de doenças, com alto índice de leishmaniose, dengue, chicungunha e Infecções Sexualmente Transmissíveis. A livre circulação na fronteira facilitava o tráfico de drogas na região.

A leishmaniose foi exemplo mencionado por vários entrevistados. Brasileiros e estrangeiros que trabalhavam em áreas de desmatamento no Peru contraíam a doença e buscavam atendimento nas UBS de Assis Brasil. A questão do tratamento medicamentoso dos estrangeiros era um dilema constante para os profissionais de saúde e para a gestão municipal, envolvendo questões de financiamento, mas também de caráter ético e humanitário. Os profissionais referiram sobrecarga assistencial em função dos atendimentos prestados aos estrangeiros que buscavam tratamento médico e odontológico no Sistema Único de Saúde (SUS), pois, em seus países, esses serviços não eram prestados por estabelecimentos públicos. Médicos brasileiros indicaram iniciativas próprias a autoridades de saúde do Peru e da Bolívia para organizar ações articuladas de prevenção e tratamento, porém sem êxito.

A busca de atendimento pelo SUS por parte de estrangeiros era uma característica comum entre os municípios da região do Alto Acre que imprimia grandes desafios às EqSF e aos gestores. 
Tabela 2. Recursos e equipamentos em saúde, Município Rural Remoto, região Norte, Assis Brasil, AC, 2019

Informações da APS

Assis Brasil (AC)

$\%$ Cobertura potencial APS

$100 \%$

Expectativa populacional 2019 (hab. $)^{2}$

7.534

População cadastrada na ESF4

8.525

$\%$ da população cadastrada e vinculada às equipes APS 4

$113 \%$

№ Equipes Estratégia da Saúde da Família’ 3

№ Equipes de Saúde Bucal ${ }^{1} \quad 3$

\begin{tabular}{ll} 
\% população beneficiária de plano de saúde ${ }^{3}$ & 0,8 \\
\hline
\end{tabular}

\section{Recursos em Saúde}

Total de no de médicos 1,5

Médico Clinico ${ }^{1}$

7

Médico da ESFI

№ Agente Comunitário de Saúde

3

№ Agente de Combate às Endemias?

№ Agente Indígena de Saneamento 1

№ Agente Indígena de Saúde`

№ Enfermeiros ${ }^{1}$

№ Assistente Social ${ }^{1}$

№ Biomédico

№ Fisioterapeuta

№ Educador Físico?

№ Farmacêutico?

№ Nutricionista ${ }^{1}$

№ Dentistas?

№ Psicólogo 1

№ Auxiliar de enfermagem ${ }^{1}$

№ Auxiliar de saúde bucal ${ }^{1}$

№ Técnico de Enfermagem ${ }^{1}$

Equipamentos de saúde ${ }^{1}$

Centro de Saúde/Unidade Básica Saúde (UBS)

UBS em áreas rurais

UBS na sede

Unidade Mista

Unidade de apoio diagnose e terapia (SADT)

Unidade móvel de suporte básico (Samu)

Farmácia

Unidade de Atenção à Saúde Indígena

Academia da saúde

Equipe de Núcleos Ampliado de Saúde da Família (Nasf)



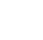

Fonte: Elaboração própria.

ISCNES: Sistema de Cadastro Nacional de Estabelecimentos, Competência, 2019.

2IBGE: Instituto Brasileiro de Geografia e Estatística, Expectativa da população, Competência, 2019.

3ANS: Agência Nacional de Saúde Suplementar, Competência, 2019.

4Sisab: Sistema de Informação em Saúde para a Atenção Básica, Painel de Cadastro, Competência, 2019.

${ }^{5}$ No município de Assis Brasil, existem duplicidades de profissionais médico no SCNES: é a situação em que um mesmo profissional está cadastrado em mais de uma função, no mesmo município. Alguns médicos estão cadastrados como 'Médico Clínico', por causa da sua atuação na Unidade Mista e, também, estão cadastrados como 'Médico da Estratégia de Saúde da Família', pela atuação nas Unidades Básicas de Saúde. 


\section{AC̣ÕES DESENVOLVIDAS PELAS EQUIPES E NO TERRITÓRIO}

Nas UBS, eram ofertados consultas, exames, vacinação, acompanhamento de grupos prioritários (crianças, mulheres, hipertensos e diabéticos), encaminhamento para atendimento especializado e visitas domiciliares. Enfermeiros e usuários ratificaram o compartilhamento dos atendimentos diários entre enfermeiros e médicos, porém, com predominância do atendimento pelos enfermeiros que realizavam encaminhamentos para o médico quando necessário. As equipes trabalhavam com programação semanal de atendimentos, assistiam à demanda espontânea e davam prioridade a usuários do interior.

Nas áreas do interior do município, as ações eram itinerantes e intermitentes. A gestão promovia ações itinerantes nas zonas rurais a fim de prestar atendimento médico, odontológico, psicológico, assistência social, vacinação e palestras. A condição climática era fator impeditivo para realização das ações itinerantes de forma mais sistemática. No período chuvoso, algumas áreas do interior ficavam inacessíveis, e no período de seca, a trafegabilidade dos rios ficava prejudicada. Essas circunstâncias levavam usuários a permanecer longos períodos (até seis meses) desassistidos. Mesmo com a promoção das ações itinerantes, residentes em áreas mais remotas do município enfrentavam dificuldades de acesso aos serviços de saúde.

As EqSF e, principalmente, os ACS também cumpriam importante papel no que tangia a ações desenvolvidas no território: instruíam sobre o consumo de água limpa e descarte adequado de lixo, distribuíam cloro para uso doméstico, sensibilizavam sobre a importância da alimentação adequada e prática de atividade física, identificavam áreas descobertas no território e usuários que precisavam de acompanhamento mais intenso. Na percepção do SMS, o ACS era a principal ponte entre os serviços de saúde e a população.

\section{DISPONIBILIDADE DE MEDICAMENTOS E REALIZAC̣ÃO DE EXAMES BÁSICOS}

A gestão centralizou a distribuição dos medicamentos para controlar a dispensação e diminuir gastos. No entanto, tal organização dificultou o acesso dos usuários que precisavam andar até uma hora meia entre uma UBS e a farmácia. Médicos flexibilizavam regras de dispensação de medicamentos para os usuários da zona rural para estoque domiciliar e relataram insuficiência, que postergava ou impedia tratamentos prescritos.

O tratamento para leishmaniose, de alta incidência na região, com média de dez casos semanais, era coordenado diretamente pela SMS; e a dispensação da medicação não acontecia sem o cartão do SUS. Alguns estrangeiros residentes em países vizinhos eram atendidos em Assis Brasil, possuíam cartão do SUS e, por meio dele, obtinham medicamentos. Mesmo para estrangeiros não portadores do cartão do SUS, eram cedidos medicamentos excedentes, mas o alto custo impossibilitava a continuidade do tratamento e gerava necessidade de mais consultas, o que aumentava a demanda pelos serviços e o tempo de espera por atendimentos. Questões relacionadas com o atendimento a estrangeiros geravam dúvidas e necessidade de mais informações para a atuação dos profissionais de saúde da APS.

A maioria dos exames realizados nas UBS de Assis Brasil e em outros municípios eram encaminhados para a capital, e o alto fluxo atrasava a chegada dos resultados. Uma usuária entrevistada que fazia acompanhamento de puerpério ainda não havia recebido os resultados de exames realizados no $3^{\circ}$ mês de gestação.

\section{COLABORAC̣ÃO INTERPROFISSIONAL E INTERSETORIALIDADE}

Em relação à colaboração interprofissional, os profissionais de saúde percebiam a atuação coesa das EqSF no compartilhamento do cuidado aos usuários. Médicos e enfermeiros 
trabalhavam em parceria no acompanhamento de usuários com agravos crônicos. ACS e técnicos de enfermagem partilhavam o acompanhamento vacinal. Técnicos da vigilância em saúde atuavam em parceria com as equipes na detecção de surtos, no acompanhamento de hanseníase e leishmaniose e na elaboração de planos estratégicos para irradicação da malária. Equipes da Fundação Nacional de Saúde (Funasa), Secretaria do Estado de Saúde e UBS compartilhavam a assistência à população indígena.

Para as ações intersetoriais, as EqSF acionavam a Secretaria de Assistência Social para prestar apoio financeiro no custeio de tratamentos ou transporte de usuários que residiam na zona rural. Gestor municipal e enfermeiro relataram o caso de uma usuária em extrema vulnerabilidade social, com transtornos psiquiátricos, cujos filhos estavam expostos a violência e privação de alimentos. Esse caso requereu a atuação conjunta da Secretaria de Saúde, das equipes do Nasf e da UBS, do Centro de Referência de Assistência Social, do Conselho Tutelar e da Prefeitura.

O Programa Saúde na Escola (PSE) foi recentemente implantado e vinha focando seus esforços na prevenção de gravidez na adolescência, crescente no município.

As ações de saúde itinerantes contavam com apoio de Organizações Não Governamentais, Secretaria de Cidadania, de Esportes, Ministério Público e entidades civis. Igrejas e escolas sediavam as atividades, e usuários cediam suas residências para hospedar os profissionais de saúde. Exército e Bombeiros ofereciam medicamentos, transporte aéreo e terrestre e atuavam em campanhas contra dengue e de vacinação.

\section{ESTRATÉGIAS PARA GARANTIA DA FORC̣A DE TRABALHO EM SAÚDE}

Os gestores não mencionaram estratégias locais para garantia da força de trabalho nos MRR. No entanto, foram enfáticos quanto à importância do Programa Mais Médicos (PMM) para a expansão da APS. O PMM garantiu a presença constante de médicos, antes apenas encontrados na unidade mista, sendo que a UBS só dispunha de equipe de enfermagem. Com a saída dos médicos cubanos, houve troca de profissionais que não permaneceram no município; e, nesse período, as UBS chegaram a ficar sete meses desassistidas. O supervisor do PMM, segundo entrevistados, mostrava-se presente e disponível para prestar apoio às EqSF.

Para profissionais e usuários, o município não oferecia boas condições de trabalho e desestimulava a fixação de profissionais na ESF. Uma nova UBS construída e equipada não pôde ser inaugurada porque não havia rede de esgoto no bairro e uma vez que a instalação elétrica era clandestina. No local, atendimentos eram prestados em uma casa adaptada que não oferecia estrutura adequada.

As UBS eram desprovidas de computador e acesso à internet, presente apenas na secretaria de saúde, o que impedia o uso do Telessaúde e e-SUS. Um enfermeiro expressou vontade de instalar uma televisão na sala de espera da UBS para exibição de vídeos educativos, sem apoio da gestão. Comunicação via telefone também não estava disponível em todo o território $\mathrm{e}$ dificultava a comunicação entre ACS e UBS. Caso este identificasse alguma emergência em campo e estivesse sem sinal de celular, precisava se locomover até a UBS, postergando o socorro.

A SMS realizava concurso público a cada dois anos para contratação de profissionais e oferecia contrato de trabalho pelo mesmo período sem possibilidade de extensão. A alta rotatividade de profissionais afetava a qualidade do serviço, causando ruptura do vínculo e da relação de confiança com os usuários, que passavam a procurar a UBS com menor frequência.

\section{ACESSIBILIDADE AOS SERVIC̣OS DE APS}

As barreiras de acesso geográfico constituíam-se obstáculos aos serviços de APS, particularmente para populações que viviam nas áreas mais distantes e de difícil acesso que 
precisavam se deslocar até a sede municipal na qual estavam concentrados os serviços de saúde. Além de longas distâncias, eram restritas as condições de mobilidade pela ausência de transporte público e pelas condições precárias das vias de acesso, principalmente no período das chuvas, quando ocorria a interdição dos ramais que interligavam o interior e a sede. Em situação de urgência, famílias chegavam a transportar usuários debilitados em redes por longo trajeto, realizado a pé, até ao ponto em que o transporte da SMS era capaz de alcançá-los. Os trajetos entre sede e interior chegavam a seis horas de deslocamento, a depender do tipo de transporte utilizado e da sazonalidade. Para as áreas indígenas, o trajeto era ainda mais longo, entre 10 e 12 horas, parte dele percorrido por via fluvial.

As dificuldades de acesso aos serviços de APS se amplificavam dadas as condições de vulnerabilidade social às quais usuários eram submetidos. Altos custos com deslocamentos, pagos pelos usuários, era importante barreira financeira que dificultava ou impedia a busca por cuidado. Aqueles que moravam em áreas distantes demoravam meses juntando dinheiro para ir até as UBS, e quando não conseguiam, caminhavam por até dois dias para chegar à sede do município. Quando tratamentos e visitas frequentes à UBS eram necessários, aqueles que moravam na zona rural e tinham condições financeiras ou familiares na sede se mudavam para a área urbana a fim de facilitar o acesso aos cuidados em saúde.

\section{SERVICOOS DE SAÚDE DE REFERÊNCIA PARA A APS}

O acesso à atenção especializada (ambulatorial e hospitalar) ocorria nos Hospitais Municipais de Brasileia e de Xapuri (Região Alto Acre), sendo Rio Branco a referência principal. Em Brasileia, havia um hospital Regional de grande porte, recém-reformado e em fase de habilitação. A contratação de médicos especialistas foi identificada pelos gestores como a principal dificuldade para o funcionamento da unidade hospitalar.
A inexistência de serviços especializados no município motivava a estreita articulação do gestor da saúde de Assis Brasil com os demais municípios da região de saúde e com a rede da capital. Para ter acesso à atenção especializada, a SMS utilizava o Sistema Nacional de Regulação (Sisreg). A gestão municipal gerenciava de perto os transportes sanitários, recurso essencial para garantir o acesso à atenção especializada, principalmente em Rio Branco. De acordo com o gestor estadual, o Ministério da Agricultura cedia vans para levar pacientes crônicos para tratamento em Rio Branco, duas vezes por semana. As viagens entre Assis Brasil e Rio Branco duravam aproximadamente seis horas. Gestores e usuários reportaram longas filas de espera para agendamento de consultas e exames na atenção especializada. Para o gestor estadual, a limitação de oferta para a atenção especializada era determinada por insuficiência de médicos especialistas no estado, por excesso de encaminhamentos feitos pela APS e por alta demanda recebida na capital, proveniente de todos os municípios.

Os usuários relataram que faziam acompanhamento médico na UBS, mas, muitas vezes, realizavam exames e tratamento especializado em serviços privados em cidades de países vizinhos, como Iñapari e San Lorenzo (Peru), na sede da região de saúde, em Brasileia, ou até mesmo na capital do estado, Rio Branco. Os principais motivos eram as longas filas de espera no SUS, os custos do deslocamento e a distância para a capital ou regional uma vez que nem sempre o transporte provido pela prefeitura era suficiente ou atendia à necessidade do usuário, além da demora na entrega dos resultados de exames. Uma usuária relatou que realizou preventivo em Assis Brasil e esperou nove meses pelo resultado. Diagnosticada a lesão, foi aconselhada por profissionais da UBS a procurar tratamento particular em cidade peruana, já utilizado por outras usuárias. Exames de imagem e serviços odontológicos não disponíveis no município também eram procurados em serviços privados no Peru ou 
na Bolívia. Profissionais das UBS encaminhavam para tratamento oftalmológico particular em Brasileia ou Rio Branco em função da alta demanda e de poucos profissionais disponíveis na rede pública.

Médicos ressaltaram a falta de recursos de Tecnologias de Informação e Comunicação em saúde que possibilitassem alguma integração com a atenção especializada, além da ausência de espaço para discussão e acompanhamento de casos. Algum tipo de contato interprofissional ocorria quando havia conhecimento pessoal prévio. Além disso, a gestão estadual informou que, na secretaria estadual, também não havia integração entre os departamentos de saúde.

Emendas parlamentares representavam importante fonte de recursos para a saúde no estado. Outrossim, parte dos recursos eram investidos em ampliação de serviços de atenção especializada, na construção e reforma das UBS e aquisição de unidades fluviais para atendimento à comunidade ribeirinha.

O quadro 2 apresenta os enunciados relevantes a partir das narrativas dos informantes-chave da pesquisa segundo as categorias analíticas.

Quadro 2. Falas expressivas dos informantes-chave segundo categorias de análise, Município Rural Remoto, Norte do Brasil, Assis Brasil, AC, 2019

\begin{tabular}{ll}
\hline Categorias & Enunciados \\
\hline Território & A gente atende essa comunidade [migrante], que não está prevista né [...]. Só que aumenta ali uma demanda que você não tem \\
& que é até fora de área [...] vem se vacinar, vem tudo aqui no Brasil. Então aí a gente tem um gasto que não tá previsto porque eles \\
& não entram na nossa cobertura populacional então, por exemplo, um município que eu tenho de 17 mil habitantes, eu acabo aten- \\
& dendo 25 mil por que tem essa migração todos os dias. Ali em Assis Brasil então é direto. (G1). \\
& Assis Brasil em si, não é só Assis Brasil, responde às cidades bolivianas e peruanas indiretamente. Pelo fato de o Brasil atender de \\
& forma gratuita é o mais buscado. (P5 MED1). \\
& 1870 indígenas abrangendo os dois rios: Rio Acre e Rio laco. Todos os programas feitos na UBS são desenvolvidos lá dentro [al- \\
& deia]: colo do útero, pré-natal, teste do pezinho, Sisvan, palestra, tudo a gente faz lá dentro. Vai com dentista também. Aí nessa \\
& época do ano, que é o inverno, nós temos muita dificuldade para desenvolver essas ações lá, devido ao acesso ao ramal; a popula- \\
& ção maior é a de lá, do Rio laco. São 1100 quase 1200 indígenas lá e a nossa dificuldade é o acesso para desenvolver essas ações, \\
& às vezes temos de ir por Sena Madureira e passam oito dias para chegar lá, para desenvolver todas as ações. Aí lá, quando a gente \\
& tem algum problema para ser notificado, algum caso novo, são todos referidos para o município. O nosso médico não pode referir \\
& diretamente para Rio Branco. É Aldeia, o município é que faz essa transferência se for necessário, para Rio Branco. (G4).
\end{tabular}

Ações desen- O serviço de vacina, imunização é espontâneo... o dia que vier manhã ou tarde vai ser atendido. Da mesma forma curativo, procevolvidas pelas dimentos injetáveis... Já aí eu vou falar a parte do médico de segunda a sexta consulta manhã e tarde. Agora enfermagem dia de equipes e no segunda manhã à tarde eu faço pré-natal. Dia de terça-feira manhã e tarde eu faço crescimento e desenvolvimento. Dia de quarta território faz preventivo manhã e tarde. Dia de quinta pelo dia manhã e tarde. Dia de sexta pré-natal pela manhã à tarde eu faço reuniões e a produção semanal e também de segunda a sexta quando o agente de saúde vem e fala da necessidade de enfermeiro e médico aí nós vamos fazer visita. (P6).

Temos um cronograma de atendimento para zona rural. O agente de saúde traz uma demanda e vamos com foco no número de famílias que moral no determinado local da zona rural. Esse ano nos fizemos um atendimento itinerante. E agora, nesse mês, temos atendimentos previstos para mais dois locais descobertos. As ações no interior duram cerca de 5 dias e contamos com o apoio das sedes das escolas e igrejas existentes no interior. (G2).

Atividades que a gente faz é visita e trabalha com orientação. A orientação é para prevenir as doenças, é coisa rotineira. Quando eu vou visitar eu peço às vezes para essa pessoa para mim ver o quintal né, aí a gente limpa né eu juntamente com morador dou uma força para tirar o lixo, os dejetos... o que eu faço é orientar né, sobre alimentação saudável no caso verdura essas coisas, eu procuro sempre orientar as pessoas a evitar fritura né e ter o hábito mais do cozido, do caldo dessas coisas. E também buscar, perguntar se está fazendo acompanhamento direitinho, vacinação [...]. (P7).

Disponibi- $\quad$ O exército que fornece medicação de vez em quando. Se você for lá no posto, a gente não tem a medicação básica na cidade. A lidade de gente fica de dois a três meses sem medicação [...] A medicação básica dificilmente tem aqui. Vem trinta dias certinho e três memedicamentos ses sem. (P8).

e realização Esse remédio é danado pra faltar. Não peguei [neste mês]. Não tinha medicamento. Aí eu tomei emprestado um cartão de credito. de exames Mas se não chegar no posto vai ser o jeito eu comprar. (U11).

básicos [Fiz os exames] na UBS. Tem exame que nem recebi ainda. Faz o exame e vai pra outro lugar, por isso ainda não recebi [...], fiz com 3 meses, mas até agora não chegou. (U12). 
Quadro 2. (cont.)

\begin{tabular}{|c|c|}
\hline Categorias & Enunciados \\
\hline $\begin{array}{l}\text { Disponibi- } \\
\text { lidade de } \\
\text { medicamentos } \\
\text { e realização } \\
\text { de exames } \\
\text { básicos }\end{array}$ & $\begin{array}{l}\text { Em fevereiro eu vim, fiz o exame, aí a enfermeira viu e não tinha nada, estava perfeito o colo do útero. Aí vão para a Secretaria, e lá } \\
\text { eles mandam para fora para poder vir o resultado. Aí eu fiz no começo de fevereiro, passou; março, abril, maio, junho, julho e não } \\
\text { chegava o resultado. Aí em novembro, eu resolvi repetir o exame porque eu ia à Secretaria e não tinha o resultado, não chegava, eu } \\
\text { não sei o que aconteceu. Aí eu repeti em novembro, e ela falou lá com a enfermeira e aí ela viu que já estava começando a lesão, aí } \\
\text { ela já me encaminhou para o ginecologista. (U16). }\end{array}$ \\
\hline $\begin{array}{l}\text { Colaboração } \\
\text { interprofissio- } \\
\text { nal intersseto- } \\
\text { rialidade }\end{array}$ & $\begin{array}{l}\text { O secretário firmou mais uma parceria não só com exército, mas exército, bombeiro todo um corpo macro de atendimento para } \\
\text { gente fortalecer a atenção, porque eles têm outro tipo de transporte, então favorece a para a gente fazer a questão de atendimento } \\
\text { nessas áreas [de difícil acesso]. A vigilância que tem muitas ONGs que trabalham numa área técnica específica de saúde mental } \\
\text { [...] nas ações de saúde itinerante é onde vai o maior número de atores, não só de ONGs mas aí entra todas as outras secretarias. } \\
\text { Entra a secretaria de cidadania, de esportes, o Ministério Público... é intersetorial. (G1). } \\
\text { O paciente com hanseníase a gente consegue compartilhar o tratamento que estão realizando, como é que tá avançando [...] o } \\
\text { pessoal do Estado vem, a coordenação, [...] nós vamos sentar com os técnicos do município aí... os diálogos são assim. (P6). }\end{array}$ \\
\hline $\begin{array}{l}\text { Estratégias } \\
\text { para garantia } \\
\text { da força de } \\
\text { trabalho em } \\
\text { saúde }\end{array}$ & $\begin{array}{l}\text { O Mais Médicos melhorou [a oferta de serviços] mesmo com a gente trabalhando com pouco [...] as pessoas sabem que tem um } \\
\text { médico na unidade. Falam até 'doutor, aqui de três a quatro anos atrás a gente tinha que conseguir uma consulta na unidade mista } \\
\text { era uma luta, porque a única médica que tinha não estava'. O posto de saúde só funcionava com a equipe de enfermagem. (P8). } \\
\text { Ficamos } 4 \text { meses sem médicos, conseguimos uma médica, mas acabou saindo, e ficamos mais } 3 \text { meses sem médicos, num total } \\
\text { de } 7 \text { meses. (G2). }\end{array}$ \\
\hline $\begin{array}{l}\text { Acessibilidade } \\
\text { aos serviços de } \\
\text { APS }\end{array}$ & $\begin{array}{l}\text { Mas em período de cheia, muitos pacientes que são ribeirinhos têm fácil acesso de barco; porque quando o rio tá seco fica mais } \\
\text { difícil, mas o que a gente tem de dificuldade de acesso é no inverno amazônico por conta da chuva, da nossa população rural, que é } \\
\text { ramal, não são pavimentados e aí fica tudo meio difícil [...]. (P9). } \\
\text { Quando chove, não tem condições de chegar nos ramais, os pacientes, às vezes, chegam carregados por redes, temos que ter } \\
\text { caminhonetes de tração para poder chegar, e muitas vezes não conseguimos, pois, o veículo acaba atolando por ter muita lama. } \\
\text { [...] Há uns dois meses fui buscar uma paciente e o carro só conseguiu chegar até certo ponto, e a paciente teve que ser carregada } \\
\text { durante duas horas para chegar até onde nós estávamos. (G2). } \\
\text { Às vezes eles dizem que há muito tempo tão precisando, mas demora mais pra juntar um dinheirinho para pagar o transporte. No } \\
\text { verão mesmo custa uma passagem 150, 50, } 80 ; 100 \text { reais que o moto taxi cobra para ir lá e trazer eles. É uma barreira financeira } \\
\text { [...]. Tem área aí pra ir de carro cobram 500, } 600 \text { reais de passagem. E o lugar mais longe que é o Picuriã, } 76 \text { km. Um carro no } \\
\text { inverno cobra } 1.000 \text { reais pra ir lá. É muito difícil o acesso. Muitos vêm a pé. Dois dias andando. (P6). }\end{array}$ \\
\hline $\begin{array}{l}\text { Serviços de } \\
\text { saúde de } \\
\text { referência para } \\
\text { a APS }\end{array}$ & $\begin{array}{l}\text { Inclusive nessa parte aí [atenção especializada] que é do estado, teve um concurso que foi feito para várias especialidades para } \\
\text { serem lotados em Brasiléia [regional], mas esses profissionais não chegaram. Existe a previsão, o negócio é chegar. (G1). } \\
\text { Em relação à atenção especializada a gente ainda tem muita dificuldade [...] é porque a gente tem número baixo de profissionais. É } \\
\text { muito escasso com relação à demanda especializada. A gente tem município aqui que não tem nenhum pediatra nem ginecologis- } \\
\text { ta. Aí tem que ser referenciado para outro município ou a capital para poder ser regulado, então é uma espera muito maior. (G3). } \\
\text { Elas pegam o transporte aqui mesmo na sede, pois o transporte não vai na zona rural, a não ser que seja caso de emergência [...] } \\
\text { fazemos viagens na madrugada de segunda-feira para terça-feira, e na madrugada de quarta-feira para quinta-feira. Saímos } 1 \mathrm{~h} \text { da } \\
\text { manhã para chegar em Rio Branco as 7h, onde serão atendidos pelos especialistas [...] são } 5 \text { a } 6 \text { horas daqui para a capital. (G2). } \\
\text { Uma estratégia para melhoria seria fortalecer as regionais com profissionais, aparelhagens etc. O que falta é planejamento [...] pela } \\
\text { falta de planejamento acontece a confusão. Não por culpa de Rio Branco, mas se eu tivesse uma regional fortalecida, não sobraria } \\
\text { tudo para Rio Branco. (G2). }\end{array}$ \\
\hline
\end{tabular}

Fonte: Elaboração própria com base nas entrevistas.

GE: Gestor Estadual | GR: Gestor Regional | GM: Gestor Municipal | ENF: Enfermeiro | MED: Médico | ACS: Agente Comunitário de Saúde | PPP: Pré-natal, Parto e Puerpério | CCU: Câncer de Colo do Útero | HAS: Hipertensão Arterial Sistêmica.

\section{Discussão}

O caso estudado, que pode ser tomado como representativo de MMR da região amazônica, apresenta um território atravessado por diversas características que adicionam à organização da APS desafios ainda mais complexos à gestão, ao processo de trabalho das equipes e ao acesso dos usuários aos serviços, entre os quais: o trânsito de estrangeiros e brasileiros pelas cidades de fronteira; a atenção às populações indígenas; a presença de áreas descobertas em 
um contexto de precárias condições de vida; a grande extensão territorial; e as condições climáticas severas ${ }^{27}$. Tais características, além da pressão assistencial por se tratar da única oferta assistencial no município, exigem das equipes aguçada competência cultural ${ }^{28}$ e condições diferenciadas de trabalho para que o cuidado possa acontecer. Ainda assim, não foram identificadas políticas e ações para a organização da APS que atenda a tais especificidades territoriais, ficando as iniciativas formais e informais a cargo do nível local, com reconhecida insuficiência e grandes dificuldades para a manutenção e sustentabilidade ${ }^{29}$.

A mobilidade transfronteiriça tem gerado grandes desafios aos gestores da saúde dos municípios do Acre e do Brasil ${ }^{30}$. O atendimento a estrangeiros e a brasileiros deve ser ponderado pelo SUS em uma perspectiva de regionalização diferenciada para esses territórios com quantitativo expressivo de população flutuante, que busca acesso à saúde onde encontra respostas mais efetivas as suas expectativas de cuidado $^{31}$. Os resultados mostram que, embora prevista na normativa, tal perspectiva de regionalização não está, de fato, implementada. Nesse contexto, profissionais da saúde enfrentam um dilema ético-profissional ante a insuficiência de recursos para atender à demanda local: não negar atendimento ao estrangeiro, tampouco deixar promover ações de combate a agravos recorrentes nos países vizinhos que geram visitas recorrentes às $\mathrm{UBS}^{\mathbf{3 2}}$.

Observa-se importante papel de políticas indutoras nacionais para a qualificação da ESF, como os Nasf, presentes e atuantes, e Academia da Saúde. Ressalta-se que, em cenários de escassa disponibilidade de equipamentos de saúde, isolamento espacial e vulnerabilidade socioeconômica, tais dispositivos podem representar a única oferta de ações voltadas às práticas promocionais de saúde, bem como apoio matricial às $\mathrm{EqSF}^{33}$, ameaçados pelo desfinanciamento federal.

Diversas foram as estratégias para ofertar APS no município, única oferta assistencial do SUS. O compartilhamento das ações entre médicos e enfermeiros, mais que desejável ${ }^{34}$, parecia ser uma necessidade imposta pelos períodos de vacância de médicos, que volta a representar um grave problema para a sustentabilidade da APS com o desmantelamento do $\mathrm{PMM}^{35}$. A enfermagem desenvolve protagonismo no cuidado aos usuários com condições crônicas, como observado em outros contextos de ruralidade ${ }^{36}$, o que deveria ser valorizado, incentivado e qualificado.

A organização das ações - principalmente, com base na demanda espontânea - é estratégica para atender a população das zonas rurais diante das imensas barreiras para o trânsito de pessoas, cujas possibilidades de deslocamento determinam o acesso aos serviços de saúde ${ }^{37}$. Dessa forma, mesmo que as ações programáticas ainda exerçam influência na forma de os profissionais organizarem seus processos de trabalho na $\mathrm{ESF}^{\mathbf{3}}$, em contexto de ruralidade, podem amplificar as inúmeras barreiras de acesso.

As ações itinerantes são dispositivos para garantia de maior acesso aos cuidados nas zonas rurais de MRR. Já o envolvimento intersetorial, assim como a colaboração interprofissional, impõe-se como estratégia de sobrevivência da APS nos territórios rurais, com otimização do uso dos recursos públicos de diversas áreas. Interiores de MRR possuem populações isoladas e com extrema dificuldade de acesso, o que não se resolve somente com a existência de UBS rurais, e vinculam-se também a determinantes sociais da saúde e a condicionalidades, como: más condições das estradas no meio rural, muitas sem pavimentação; escassez de transporte para a locomoção das EqSF às localidades rurais; falta de transporte público; precário acesso aos meios de comunicação; e alta rotatividade de profissionais da saúde, em especial, o médico ${ }^{39}$. De toda forma, a continuidade das iniciativas itinerantes dependia de investimentos em meios de transporte adequados para o deslocamento dos profissionais, essencial nesses cenários ${ }^{40}$. Em um país desigual, continental, federativo como o Brasil, é necessário o reconhecimento 
das piores condições de vida e de mobilidade que caracterizam os cenários de ruralidade, que exigem a ação articulada de vários setores da política, não efetivamente atendidas por políticas distritais/municipais. Nessa perspectiva, um dos desafios para a formulação de políticas de saúde para MRR é o necessário equilíbrio entre o reconhecimento das realidades e das iniciativas locais e o necessário protagonismo dos entes federal e estadual na condução e no financiamento.

A despeito dos esforços identificados para a organização e a manutenção da APS no território, a insuficiência de medicamentos básicos, em contexto de pobreza e de grande dependência dos programas de transferência de renda, representa um duro golpe para a resolutividade do cuidado na APS40,41. Além de aumentar a pressão assistencial sobre as equipes, amplia as desigualdades em saúde ao condicionar a aquisição de remédios à capacidade individual de pagamento ${ }^{\mathbf{4 1}}$.

As dificuldades de trafegabilidade, em parte, são determinadas pelas condições climáticas de áreas de floresta tropical ${ }^{\mathbf{4 2}}$. Contudo, em geral, MRR são também áreas de menor dinamismo econômico e pior acesso ao conjunto de bens públicos, como saneamento, energia elétrica, políticas habitacionais e condições das estradas ${ }^{\mathbf{4 3}}$. Nesse sentido, apartado de uma concepção de desenvolvimento amplo e inclusivo para tais territórios, as iniciativas do setor saúde tornam-se parciais e incompletas, exigindo de profissionais, usuários e gestores locais a busca de soluções individuais e esparsas.

Apesar das inúmeras inciativas para utilização dos recursos locais em saúde, a integração e a regionalização dos serviços na região, não diferente de outros cenários ${ }^{\mathbf{4 4}}$, eram insuficientes para atender à demanda por retaguarda terapêutica. Não obstante, parecia haver recursos especializados privados na região, indicado por profissionais na falta de perspectiva de consecução pelo SUS ${ }^{45}$, ou seja, mesmo se tratando de um MRR, não havia completa ausência de serviços especializados, mas indisponibilidade destes via oferta pública. Duas questões parecem ser prementes, certamente não apenas em contexto rural remoto, mas imprescindíveis nesses cenários: fortalecer as regiões de saúde para que cumpram a função de oferta de atenção ambulatorial especializada44,46; e oferta irrestrita de Tecnologias de Informação e Comunicação em Saúde como mediadores e qualificadores do cuidado, tanto para profissionais quanto para usuários, a exemplo do que já acontece em MRR de muitos países ${ }^{47}$.

Como limitação do estudo, menciona-se que a análise apresentada se circunscreve a elementos do sistema formal de organização da APS, não considerando outros aspectos que se relacionam com os subsistemas informal e popular, também determinantes para uma compreensão mais ampliada do processo saúde-doença nesses territórios. Foram destacados os elementos mais relevantes na experiência e na percepção dos participantes da pesquisa que não encerram o conjunto de componentes organizacionais da APS, ainda que sinalizem seus principais êxitos e desafios a partir da experiência singular de Assis Brasil.

\section{Considerações finais}

Elementos relacionados com a saúde internacional devem ser considerados na organização da APS em localidades de fronteira, já que a linha fictícia que separa o território nacional não é suficiente para interromper relações sociais, culturais e econômicas que determinam a saúde no território. Em relação à atenção aos indígenas, o caso de Assis Brasil revela a necessária interação entre as políticas específicas para os povos indígenas e o SUS, sob pena de prevalecerem a desassistência, a inadequação e a incompletude do cuidado. A competência cultural, no caso de Assis Brasil, assume posição de atributo essencial da APS, quando à multiculturalidade é à particularidade do contexto. 
Argumenta-se que MRR e suas populações somam vulnerabilidades econômicas, sociais e de acesso aos serviços de saúde, parcialmente atendidas pelas políticas nacionais, e que o ente municipal, sem o suficiente apoio e aporte de recursos estadual e federal, mantém arranjos possíveis para a provisão de APS, nem sempre afeitas aos princípios abrangentes que caracterizam a ESF. O estudo ratifica a incontestável necessidade de manutenção e aperfeiçoamento de políticas federais e estadual para provisão e formação de médicos para atuação em MRR; políticas de financiamento para o transporte sanitário; capacitação e insumos farmacológicos para que EqSF possam assumir maior protagonismo e resolutividade das ações; manutenção do financiamento federal das equipes de Nasf; implementação do telessaúde em suas diversas funcionalidades; valorização do escopo ampliado de práticas de enfermeiros e ACS; associados à premente necessidade de fortalecer arranjos regionais para oferta de retaguarda terapêutica em tempo oportuno. Ressalta-se que investimentos no setor saúde devem ser acompanhados por melhorias nas condições de vida.

Os achados do estudo reafirmam a necessidade de adaptação das políticas públicas às realidades sociossanitárias dos territórios e populações rurais com vistas a, no campo da saúde, garantir o direito à atenção integral, universal e igualitária no SUS.

\section{Agradecimentos}

Almeida PF é Bolsista de Produtividade do Conselho Nacional de Desenvolvimento Científico e Tecnológico (CNPq) e agradece o apoio recebido para o desenvolvimento de estudos sobre o tema.

\section{Colaboradores}

Rodrigues KV (0000-0002-3493-8617)* foi responsável pela análise, interpretação dos resultados e redação do manuscrito. Almeida PF (0000-0003-1676-3574)*, Cabral LMS (0000-0001-6144-8050) e Fausto MCR (00000003-0746-3684)* foram responsáveis pela concepção, interpretação dos resultados, redação e revisão do manuscrito. Fausto MCR coordenou o estudo 'Atenção Primária à Saúde em territórios rurais e remotos no Brasil'. 


\section{Referências}

1. Zhao Y, Thomas SL, Guthridge SL, et al. Better health outcomes at lower costs: the benefits of primary care utilisation for chronic disease management in remote Indigenous communities in Australia's Northern Territory. BMC Health Serv Res. 2014 [acesso em 2020 nov 10]; 14:463. Disponível em: http://www. biomedcentrl.com/1472-6963/14/463.

2. Wakerman J, Humphreys J, Russell D, et al. Remote health workforce turnover and retention: what are the policy and practice priorities? Hum Resour Health. 2019; 17:99.

3. World Health Organization. The Global health observatory. World Health Data Platform /GHO /Themes. Urban health. [acesso em 2020 nov 1]. Disponível em: https://www.who.int/data/gho/data/themes/ theme-details/GHO/urban-health.

4. Portela GZ, Fehn AC, Ungerer RLS, et al. Recursos humanos em saúde: crise global e cooperação internacional. Ciênc. Saúde Colet. 2017; 22(7):2237-2246.

5. Wakerman J, Bourke L, Humphreys JS, et al. Is remote health different to rural health? Rural Remote Health. 2017; 17(2):3832.

6. Strasser R. Learning in context: education for remote rural health care. Rural Remote Health 2016; 16:4033.

7. Scheil-Adlung X. Global evidence on inequities in rural health protection: new data on rural deficits in health coverage for 174 countries. 2015; ESS Doc. 47, International Labour Organization. Geneva: ILO; 2015. [acesso em 2020 nov 2]. Disponível em: http:// www.social-protection.org/gimi/gess/RessourcePDF. action?ressource.ressourceId $=51297$.

8. Ferreira MJM. Políticas públicas de saúde para as populações do campo: avanço e desafios para sua efetivação. In: Almeida DG, Viana Júnior MM, Chaves RLSC, et al., organizadores. História, memória e conflitos territoriais no Ceará: comunidades do Tabuleiro de Russas. Fortaleza: EdUECE; 2019. p. 151159.
9. Instituto Brasileiro de Geografia e estatística. Cidades. Censo demográfico 2010. IBGE; 2017. [acesso em 2021 out 2]. Disponível em: https://cidades.ibge.gov. br/2017.

10. Brasil. Ministério da Saúde. Departamento de Informática do Sistema Único de Saúde. 2017. [acesso em 2021 out 2]. Disponível em: https://datasus.saude. gov.br/.

11. Le Tourneau FM. Sparsely populated regions as a specific geographical environment. J Rural Stud. 2020; 75:70-79.

12. Strasser R, Worley P, Cristobal F, et al. Putting communities in the driver's seat: the realities of community engaged medical education. Academic Medicine. 2015; (90):1466-1470.

13. Behera MR, Prutipinyo C, Sirichotiratana N, et al. Interventions for improved retention of skilled health workers in rural and remote areas. Ann Trop Med Public Health. 2017; 10(1):16-21.

14. Zhang X, Badee uz Z. Adoption mechanism of telemedicine in underdeveloped country. Health Informatics. 2020; 26(2):1088-1103.

15. Macinko J, Harris MJ. Brazil's family health strategy-delivering community-based primary care in a universal health system. New Engl J Med. 2015; 372(23):2177-81.

16. Santos LMP, Oliveira A, Trindade JS, et al. Implementation research: towards universal health coverage with more doctors in Brazil. Bull World Health Organ. 2017; 95(2):103-12.

17. Instituto Brasileiro de Geografia e Estatística. Classificação e caracterização dos espaços rurais e urbanos do Brasil: uma primeira aproximação. Brasília, DF: IBGE; 2017. [acesso em 2020 set 17]. Disponível: https://biblioteca.ibge.gov.br/visualizacao/livros/ liv100643.pdf. 
18. Stake R. The art of case study research. Thousand OAKS: Sage; 1995. p. 49-68.

19. Fausto MCR, Cabral LMS, Braz HMR, et al. Assis Brasil, AC: Relatório final de pesquisa. Rio de Janeiro: Fiocruz; 2020.

20. Instituto Brasileiro de Geografia e Estatística. Indígenas: Gráficos e tabelas. Tabela Municípios com as maiores proporções de população indígena do País, por situação do domicílio, Acre. Brasília, DF: IBGE; 2020. [acesso em 2020 ago 20]. Disponível em: https://indigenas.ibge.gov.br/graficos-e-tabelas-2.html.

21. Kessner DM, Kalk CE, Singer J. Assessing health quality - The case for tracers. New Engl J Med. 1973; 288(4):189-94.

22. Fausto MCR, Oliveira AC, Bousquat A, et al. Atenção Primária à Saúde em territórios rurais e remotos no Brasil: Projeto de Pesquisa. Rio de Janeiro: Fiocruz; 2019.

23. Minayo MCS. O desafio do conhecimento: Pesquisa Qualitativa em Saúde. 12. ed. São Paulo, SP: Hucitec-Abrasco; 2010.

24. Almeida PF, Medina MG, Fausto MCR, et al. Coordenação do cuidado e Atenção Primária à Saúde no Sistema Único de Saúde. Saúde debate. 2018; 42(esp1):244-260.

25. Bousquat A, Giovanella L, Fausto MCR, et al. A atenção primária em regiões de saúde: política, estrutura e organização. Cad. Saúde Pública 2019; 35(supl2):e00099118.

26. Wakerman J, Humphreys JS, Wells R, et al. Primary health care delivery models in rural and remote Australia - a systematic review. BMC Health Serv. 2008; (8):276.

27. Soares RAS, Moraes RM, Vianna RPT, et al. Determinantes socioambientais e saúde: o Brasil rural versus o Brasil urbano. Tempus. 2015; (9):221-35.
28. Damasceno RF, Silva PLN. Competência cultural na atenção primária: algumas considerações. J Manag Prim Health Care. 2018 [acesso em 2021 jan 29]; (90). Disponível em: https://jmphc.com.br/jmphc/article/ view/435.

29. Garnelo L. Especificidades e desafios das políticas públicas de saúde na Amazônia. Cad. Saúde Pública. 2019; 35(12):e00220519.

30. Cazola LHO, Pícoli RP, Tamaki EM, et al. Atendimentos a brasileiros residentes na fronteira Brasil-Paraguai pelo Sistema Único de Saúde. Rev Panam Salud Publica. 2011; 29(3):185-190.

31. Lemões MMA, Lange C, Castro P, et al. Programa mais médicos na fronteira: gestão em saúde em cidades gêmeas entre Brasil e Uruguai. Rev Urug Enferm. 2019; 14(1):38-48.

32. Costa EMS, Costa EA, Cunha RV. Desafios da prevenção e controle da dengue na fronteira Brasil/Bolívia: representações sociais de gestores e profissionais da saúde. Physis. 2019; 8(04):e280415.

33. Costa LA, Carneiro FF, Almeida MM, et al. Estratégia Saúde da Família rural: uma análise a partir da visão dos movimentos populares do Ceará. Saúde debate. 2019; 43(esp8):36-49.

34. Farias DN, Ribeiro KSQS, Anjos UU, et al. Interdisciplinaridade e interprofissionalidade na estratégia saúde da família. Trab Educ Saúde. 2017; 16(1):141162.

35. Giovanella L, Bousquat A, Almeida PF, et al. Médicos pelo Brasil: caminho para a privatização da atenção primária à saúde no Sistema Único de Saúde? Cad. Saúde Pública. 2019; 35(10):e00178619.

36. Rosa BM, Silva BT, Sousa JIS, et al. Profile of the elderly resident of the rural area and the use of medicines. RSD. 2020; 9(10):e589108292.

37. Garnelo L, Lima JG, Rocha ESC, et al. Acesso e cobertura da Atenção Primária à Saúde para popula- 
ções rurais e urbanas na região norte do Brasil. Saúde debate. 2018; 42(esp1):81-99.

38. Brasil. Ministério da Saúde. Portaria n ${ }^{\circ}$ 2.436, de 21 de setembro de 2017. Aprova a Política Nacional de Atenção Básica, estabelecendo a revisão de diretrizes para a organização da Atenção Básica, no âmbito do Sistema Único de Saúde (SUS). Diário Oficial da União. 21 Set 2017.

39. Pessoa VM, Almeida MM, Carneiro FF. Como garantir o direito à saúde para as populações do campo, da floresta e das águas no Brasil? Saúde debate. 2018; 42(espl): 302-314.

40. Almeida LC, Souto MK, Nogueira GAMH, et al. Qualidade do cuidado: Avaliação da disponibilidade de insumos, imunobiológicos e medicamentos na Atenção Básica em município de Minas Gerais, Brasil. Rev Bras Med Fam Comunidade. 2019 [acesso em 2021 jan 29]; 14(41): 1900. Disponível em: https://rbmfc org.br/rbmfc/article/view/1900.

41. Pereira LL, Pacheco L. O desafio do Programa Mais Médicos para o provimento e a garantia da atenção integral à saúde em áreas rurais na região amazônica, Brasil. Interface. 2017; 21(supl1):1181-1192.

42. Guimarães AF, Barbosa VLM, Silva MP, et al. Acesso a serviços de saúde por ribeirinhos de um município no interior do estado do Amazonas, Brasil. Rev Pan-Amaz Saude. 2020; 11:e202000178.
43. Arruda NM, Maia AG, Alves LC. Desigualdade no acesso à saúde entre as áreas urbanas e rurais do Brasil: uma decomposição de fatores entre 1998 a 2008. Cad. Saúde Pública. 2018; 34(6):e00213816.

44. Fausto MCR, Almeida PF, Bousquat A. Organização da atenção primária à saúde no Brasil e os desafios para integração em redes de atenção. In: Mendonça MHM, Matta GC, Gondim R, et al. Atenção Primária à Saúde no Brasil: conceitos, práticas e pesquisa. Rio de Janeiro: Ed. Fiocruz; 2018. p. 51-72.

45. Almeida PF. Atenção primária à saúde no Brasil e os 40 anos de Alma-Ata: reconhecer os desafios para seguir adiante. Cad. Saúde Pública 2018; 34(8):e00136118.

46. Almeida PF, Giovanella L, Martins FMT, et al. Redes regionalizadas e garantia de atenção especializada em saúde: a experiência do Ceará, Brasil. Ciênc. Saúde Colet. 2019; 24(12):4527-4540

47. Harper K, McLeod M, Brown SK, et al. Teleneurology service provided via tablet technology: 3-year outcomes and physician satisfaction. Rural Remote Health. 2019; 19(1):4743.

Recebido em 22/03/2021

Aprovado em 06/10/2021

Conflito de interesses: inexistente

Suporte financeiro: Ministério da Saúde por meio do Termo de Cooperação 085/2015 no âmbito do Programa Nacional de Melhoria do Acesso e da Qualidade da Atenção Básica 